\title{
In defence of consent and capacity boards for end-of-life care
}

\author{
Jocelyn Downie, SJD • Michael Hadskis, LLM
}

Received: 3 June 2014/ Accepted: 18 July 2014/Published online: 26 August 2014

(c) Canadian Anesthesiologists' Society 2014

In Cuthbertson v. Rasouli, the Supreme Court of Canada (SCC) found that, in Ontario, it is the Consent and Capacity Board (CCB) and not the courts per se who will resolve conflicts between substitute decision-makers (SDMs) and health practitioners regarding the withdrawal of lifesustaining treatment from incapable patients. This finding was based on the SCC's interpretation of the Ontario Health Care Consent Act (HCCA). Hawryluck et al. express concern about the SCC's determination that the $\mathrm{CCB}$ is charged with resolving such conflicts since, in their view, this body is ill-equipped to fulfill this role. ${ }^{1}$ Instead, they take the position that these disputes should be adjudicated by the courts. We disagree with this position and, for the reasons set out in this editorial, take the position that provincial and territorial legislators across the country should follow the lead of the Ontario legislature, revise their health care consent legislation to clarify the law with respect to the unilateral withholding and withdrawal of potentially life-sustaining treatment, and establish open and transparent consent and capacity tribunals to deal with irreconcilable conflicts in this context.

\section{Arguments made against the CCB}

Hawryluck et al. raise a number of "limitations" of the $\mathrm{CCB}$, including ones related to expertise, mandate and means, as well as timeliness. Most of them, we argue, are not sustainable.

J. Downie, SJD $(\bowtie) \cdot$ M. Hadskis, LLM

Schulich School of Law, Dalhousie University, 6061 University

Avenue, PO Box 15000, Halifax, NS B3H 4R2, Canada

e-mail: Jocelyn.Downie@Dal.Ca
With respect to expertise, they take issue with the composition of the $\mathrm{CCB}$ on the basis that: "The CCB currently has no specialty critical care representation among its members and does not seek to develop expertise by having the same members hear critical care cases. These drawbacks mean that deliberations of the CCB may lack specific expertise of content and sufficient context and breadth of opinion to adjudicate whether a SDM is acting in the 'best interests' of a critically ill patient (HCCAs 21(2)), especially with respect to any medical benefits and risks of the proposed or ongoing lifesustaining treatments in question."

While it is true that the CCB does not currently have members with critical care expertise, it should be pointed out that neither do the courts. In any event, we agree that it would be worthwhile to enlist physicians with expertise in end-of-life care to these boards. However, as opposed to Hawryluck et al., we do not confine the appropriate experts to critical care specialists - there are other medical specialties that would also be well-suited to contribute to the adjudication of conflicts regarding end-of-life treatment, including specialists in palliative care.

In addressing the issues of mandate and means, Hawryluck et al. contend that the CCB does not have sufficient "mandate or means to deliberate questions regarding the standard of care and where a treatment falls outside of the standard of care. Physicians must prove to the panel that withdrawing the treatment is in the 'best interests' of the patient." In our view, the CCB has the statutory mandate to resolve the kinds of disputes that arise between SDMs and health practitioners regarding the withdrawal of life-sustaining treatment. When a health practitioner wishes to challenge the SDM's decision, s/he can ask the CCB to determine whether the SDM complied with section 21 of the Ontario HCCA, which requires the 
SDM to consider, among other things, whether the treatment would medically benefit the patient. ${ }^{2}$ Thus, the $\mathrm{CCB}$ is required to consider medical evidence respecting the patient's condition and prognosis, the expected result of treatment for the patient, and any risks of treatment. Therefore, the HCCA clearly authorizes and requires the CCB to make best interest determinations on the basis of all relevant facts put before it, including medical evidence concerning the presence or absence of medical benefit associated with the life-sustaining treatment in question.

In terms of whether the $\mathrm{CCB}$ has the means to fulfill their mandate, the majority of the SCC concluded that the "Board is well placed to make a determination of whether treatment is in the best interests of the patient, in light of the statutory objectives of enhancing patient autonomy and ensuring appropriate medical care" ${ }^{3}$ and that "[o]ver the past 17 years, the Board has developed a strong track record in handling precisely the issue raised in [the Rasouli] case."4 The SCC's opinion in this regard is supported by the Ontario Court of Appeal's decision in M. (A.) v. Benes, which observed that a "disagreement between the S.D.M. and the health practitioner potentially creates tension and the [HCC] Act recognizes this by providing for a neutral expert board to resolve the disagreement. Indeed, after hearing submissions from all parties, the Board is likely better placed than either the S.D.M. or the health practitioner to decide what is in the incapable person's best interests." ${ }^{5} \mathrm{We}$ agree with the Supreme Court of Canada and the Ontario Court of Appeal; the CCB is well placed to adjudicate the issue of whether withdrawal of treatment is in the best interests of the patient.

The timeliness of the CCB's adjudicative process was also an issue criticized by Hawryluck et al.: ${ }^{1}$

"The timeliness of the CCB process is also problematic. In contrast to what has been reported in the media, the physician authors' personal experiences in such adjudications maintain that such hearings are often inefficient and prone to delays and month-long adjournments. Rulings may be appealed to the Superior Court, which could take months to schedule a hearing. Superior Court rulings may then be appealed to the Court of Appeal, which again could take months with no statutory limit on the time frame to render a decision."

Although "personal experience" is offered by the physician authors in support of their criticism regarding timeliness, no direct evidence is provided to support their argument. In terms of the timeliness of the CCB's decision-making process, the CCB's Service Standard Policy states: ${ }^{6}$

"The Health Care Consent Act sets out three legislated service standards for the CCB:
1. A hearing is to commence within seven (7) days from the receipt of an application.

2. The CCB must issue its Decision within one day of the conclusion of the hearing.

3. Upon the request of a party, the CCB must issue written Reasons for its Decision, within four business days of receiving such a request."

Indeed, a systematic review of the end-of-life cases decided by the CCB revealed that the average length of time from the hearing beginning to a final decision from the CCB was less than 12 days, with a median time of two days. Furthermore, almost half of all decisions were released within 24 hours of the initial hearing. Some cases were decided on the same day of the hearing, while the longest delay from the beginning of the hearing to the CCB decision was 93 days. $^{7}$

Furthermore, while the authors' statement concerning delays associated with appeal processes is true, it fails to recognize that going to court ( $v s$ making an application to the tribunal) in the first instance will have the same subsequent time delays with respect to appeals. The only relevant comparison is tribunal time to "court of first instance" time and, on that comparison, the CCB is preferable. Thus, the authors' criticism regarding the timeliness of the CCB's adjudicative process is not well supported.

\section{Arguments in support of a CCB-like process}

The SCC explicitly addressed the superiority of tribunals, such as the CCB, over the courts for the resolution of conflict in the context of withholding and withdrawal of potentially life-sustaining treatment. In responding to the physicians' position in Rasouli that the court is the appropriate venue, Chief Justice McLachlin wrote: ${ }^{8}$

"...I am concerned about the practical impact of the proposed approach on patients, families, and physicians. It may heighten the vulnerability of incapable patients, since the legal burden will be on family or friends to initiate court proceedings to prevent the withdrawal of life support, rather than on physicians to obtain consent before acting. The implications of this shift are particularly troubling where the incapable patient lacks a network of family and friends with the financial resources to fund legal action, which could entail a trial on the medical standard of care. Furthermore, it is unclear on the proposed approach whether the physician could withdraw life support without judicial oversight if no legal action is taken by the patient's family or friends. 
One of the legislature's primary motivations in enacting the HCCA was to simplify the law governing the treatment of incapable patients. The HCCA sets out clear rules requiring consent before treatment can occur, identifying who can consent for an incapable patient, stating the criteria on which consent must be granted or refused, and creating a specialized body to settle disputes. The legal framework of the HCCA has been used to resolve end-of-life disputes in Ontario for 17 years. I would be reluctant to close off access to this established regime and cast these matters back into the courts."

Indeed, it is widely recognized that tribunals offer great advantages over the courts. In a recent speech, the Chief Justice of the SCC stated that: ${ }^{9}$

"Tribunals provide specialized and technical resolutions in different situations, ensure greater innovation, flexibility and efficiency in the delivery of governmental programs and strategies, and provide an informal and rapid forum for public hearings, thereby minimizing time and costs related to litigation before ordinary courts."

Accordingly, the Ontario $\mathrm{CCB}$ process represents an improvement over all other provincial/territorial health care conflict resolution processes, all of which rely on court-based adjudication. The CCB process is less formal than court processes and thus is likely less daunting and intimidating for the parties involved. Furthermore, the CCB is not obligated to follow the stricter rules of evidence adhered to by the courts that can make hearings formal and highly technical. ${ }^{10}$ Lastly, the CCB requires that the health practitioners themselves (rather than the SDM) initiate the CCB application and, having done so, discharge the burden of proving that the SDM did not comply with section 21 of the Ontario HCCA. Hawryluck et al. recommend that SDMs be required to petition the court for an injunction and to meet the applicable burden of proof that would be placed on them to obtain such relief - this could be a substantial obstacle for SDMs lacking the financial and emotional wherewithal to engage with the courts in this manner.

Though no tribunal is perfect, we would argue that the advantages of the CCB dispute resolution processes outweigh the weaknesses and that other jurisdictions should look to Ontario for guidance, reform their health care consent legislation, and introduce tribunals for the resolution of conflicts over consent and capacity.

Hawryluck et al. conclude that "[t]he Cuthbertson v. Rasouli decision is best read as a call to the critical care field to reflect on and define clearly its role in the care of patients. Most importantly we must explain to patients and
SDMs the circumstances when we may be able to help and when we cannot, no matter how much we as healthcare providers may yearn to do so." We would argue, however, that the Rasouli decision is better read as a call to people throughout Canada to demand better of their governments and to expect law reform to ensure that physicians do not have (or believe themselves to have) the authority to unilaterally withhold or withdraw potentially life-sustaining treatment. As manifest in Ontario through this issue, the touchstones should be respect for patient autonomy and a rebuttable presumption that SDMs have a greater capacity than critical care physicians to determine what is in the best interests of their loved one. Prima facie decision-making authority should rest with patients or their SDMs with conflicts to be resolved by a CCB.

\section{Les soins de fin de vie: pour une commission du consentement et de la capacité}

Dans l'affaire Cuthbertson contre Rasouli (Rasouli), la Cour suprême du Canada (CSC) a estimé qu'en Ontario, c'était à la Commission du consentement et de la capacité (CCB) et non aux tribunaux en tant que tels de résoudre les conflits entre les mandataires et les professionnels de la santé concernant l'arrêt d'un traitement nécessaire au maintien de la vie chez des patients inaptes. Cette décision se basait sur l'interprétation, par la CSC, de la loi Health Care Consent Act (HCCA; loi ontarienne sur le consentement aux soins de santé). Hawryluck et coll. expriment cependant leurs inquiétudes quant à cette décision de la CSC qui attribue au CCB la responsabilité de résoudre de tels conflits. En effet, selon eux, cet organisme est peu préparé à remplir ce rôle. ${ }^{1}$ Hawryluck et coll. croient que ce type de litiges devraient être tranché par les tribunaux. Nous ne sommes pas d'accord avec ce point de vue, étant plutôt d'avis que les législateurs provinciaux et territoriaux de tout le pays devraient suivre l'exemple de l'Ontario et revoir leur législation sur le consentement aux soins de santé afin de clarifier les lois concernant le maintien ou le retrait unilatéral d'un traitement nécessaire au maintien de la vie. Tout comme en Ontario, nous encourageons la mise place de commissions ou tribunaux compétents en matière de consentement et d'aptitude, ouverts et transparents, ayant pour tâche de résoudre les conflits irréconciliables.

\section{Arguments avancés contre le CCB}

Hawryluck et coll. soulèvent certaines «limites » inhérentes au CCB. Celles-ci touchent l'expertise, le 
mandat et les moyens, ainsi que la possibilité d'agir en temps opportun. La plupart d'entre elles ne sont pas valides à nos yeux.

Sur le plan de l'expertise, Hawryluck et coll. voient un problème dans la composition du CCB en se basant sur le fait que: «le $\mathrm{CCB}$ ne compte actuellement aucun spécialiste en soins intensifs parmi ses membres et qu'aucun membre n'est sollicité de manière régulière dans les cas impliquant les soins intensifs, ce qui permettrait l'acquisition d'une meilleure expertise sur le sujet. Ces inconvénients signifient qu'il est possible que l'expertise touchant spécifiquement le contenu et le contexte, apte à procurer une perspective suffisamment large lors des délibérations du CCB visant à examiner si un mandataire agit dans le "meilleur intérêt" d'un patient dans un état critique, s'avère insuffisante (HCCA, art. 21[2]). Ceci est particulièrement vrai lorsqu'il est question des bénéfices et risques médicaux liés aux traitements proposés ou en cours. »

S'il est exact que le CCB ne compte pas actuellement de membre ayant une expertise en soins intensifs, il faut noter que les tribunaux n'en ont pas davantage. D'une façon générale, sous sommes d'accord qu'il serait utile que des médecins ayant une expertise en soins de fin de vie soient recrutés par des commissions de type CCB. Cependant, contrairement à Hawryluck et coll., nous ne croyons pas que les intensivistes soient les seuls experts compétents dans ce domaine: d'autres spécialités médicales seraient également bien placées pour contribuer aux jugements sur les conflits touchant les traitements de fin de vie, y compris des spécialistes en soins palliatifs.

Hawryluck et coll. soutiennent que le CCB ne possède " ni le mandat, ni les moyens pour délibérer sur des questions concernant les normes de soins et/ou pour juger si un traitement se situe en dehors de celles-ci. Les médecins doivent démontrer au membres de la Commission que l'arrêt du traitement est dans le meilleur intérêt du patient ». Selon nous, le CCB a le mandat statutaire de résoudre les conflits survenant entre les mandataires et les professionnels de la santé concernant l'arrêt d'un traitement nécessaire au maintien de la vie. Lorsqu'un praticien souhaite contester la décision d'un mandataire, il peut demander au CCB de déterminer si le mandataire s'est conformé à l'article 21 de la loi HCCA de l'Ontario qui demande au mandataire de considérer - entre autres choses - l'éventuel bénéfice médical du traitement pour le patient. ${ }^{2}$ Ainsi, le CCB est tenu de prendre en compte les constatations médicales concernant l'état et le pronostic du patient, le résultat escompté du traitement et tous les risques qui lui sont attachés. Donc, la HCCA autorise et demande clairement au CCB de déterminer quel est le meilleur intérêt du patient sur la base de tous les faits pertinents qui lui sont présentés, y compris les données médicales attestant de l'existence ou de l'absence d'un bénéfice médical associé au traitement de maintien de vie dont il est question.

Pour ce qui est de savoir si le CCB a les moyens de remplir son mandat, la majorité des juges de la CSC a conclu que la «Commission était bien placée pour prendre une décision quant à savoir si le traitement allait dans l'intérêt du patient, en considérant les objectifs statutaires qui sont l'amélioration de l'autonomie du patient et l'importance de recevoir des soins médicaux appropriés $»^{3}$; la Cour a également conclu qu'au cours « des 17 dernières années, la Commission a développé une solide réputation concernant précisément la gestion des problèmes, comme celui soulevé dans le cas Rasouli. » ${ }^{4}$ L'avis de la CSC sur ce point est appuyé par une décision de la Cour d'appel de l'Ontario dans l'affaire $M$. (A.) contre Benes, qui a fait remarquer qu'un « désaccord entre le mandataire et le professionnel de la santé peut créer des tensions et que la loi (HCCA) le reconnaît en procurant un conseil d'experts neutres pour résoudre le désaccord. De fait, après avoir entendu les conclusions de toutes les parties, la Commission est probablement mieux placée que le mandataire ou le professionnel de la santé pour décider de ce qui va dans le meilleur intérêt de la personne inapte. ${ }^{5}$ Nous sommes d'accord pour dire que le CCB est mieux placé que les tribunaux pour trancher la question de savoir si l'arrêt d'un traitement va dans le meilleur intérêt du patient.

La possibilité, pour le $\mathrm{CCB}$, de rendre des décisions dans un temps opportun a également été critiquée par Hawryluck et coll: ${ }^{1}$

«La rapidité du processus décisionnel du CCB est également problématique: contrairement à ce qui a été dit dans les médias, notre expérience personnelle est que ces audiences sont souvent inefficaces et sujettes à des retards et à ajournements prolongés. Il est possible de faire appel des décisions en Cour supérieure, où l'obtention d'une audience peut prendre des mois. Il est ensuite possible de faire appel des décisions de la Cour supérieure auprès de la Cour d'appel, ce qui - de nouveau - prend des mois, sans obligation statutaire de rendre une décision dans période déterminée. »

Même si les auteurs font état de leur "expérience personnelle" pour soutenir leurs critiques concernant la vitesse du processus, ils n'apportent aucune preuve directe pour supporter leur argument. En ce qui concerne le temps nécessaire aux processus décisionnels du CCB, sa politique normative indique que: ${ }^{6}$

"La loi HCCA établit trois normes de services régissant le $\mathrm{CCB}$ : 
1. Une audience doit commencer dans les sept (7) jours suivant la réception d'une demande.

2. Le CCB doit rendre sa décision dans les 24 heures suivant la fin de l'audience.

3. Sur demande d'une des parties, le CCB doit fournir par écrit les motifs de sa décision dans les quatre jours ouvrables suivant la réception d'une telle demande."

Et, de fait, une revue systématique des cas de fin de vie ayant fait l'objet d'une décision par le CCB a révélé un délai moyen inférieur à 12 jours entre le début de l'audience et la décision rendue, avec un délai médian de deux jours. De plus, presque la moitié de toutes les décisions ont été rendues dans les 24 heures suivant l'audience initiale; quelques cas ont fait l'objet d'une décision le jour même. Dans un cas, Le plus long délai observé entre le début des audiences et la décision du CCB a été de 93 jours. ${ }^{7}$

De plus, même si l'affirmation des auteurs concernant les délais associés aux procédures d'appel est vraie, il convient de noter qu'emprunter d'emblée la voie des tribunaux (contre déposer une requête devant un tribunal d'arbitrage ou une commission) entraînera les mêmes délais dans l'éventualité d'un appel. La seule comparaison pertinente est celle du délai d'obtention d'une audience devant la Commission et devant une cour: à ce titre, le CCB est préférable. Ainsi, la critique des auteurs concernant les délais associés au processus de décision du CCB n'est pas bien étayée.

\section{Arguments en faveur d'une procédure de type CCB}

La CSC a explicitement abordé la question de la supériorité des tribunaux d'arbitrage ou commissions tels que le CCB par rapport aux tribunaux réguliers pour la résolution de conflits touchant le retrait, ou non, d'un traitement potentiellement nécessaire au maintien de la vie. En réponse au point de vue des médecins selon lesquels la Cour est l'instance la plus appropriée pour juger l'affaire, la Juge en chef McLachlin a écrit (dans le cas Rasouli): ${ }^{8}$

«...Je suis préoccupée par l'impact pratique de l'approche proposée pour les patients, les familles et les médecins. Cela pourrait accroître la vulnérabilité de patients inaptes dans la mesure où il incombera à la famille ou aux amis d'assumer le fardeau d'entamer les procédures devant un tribunal pour empêcher l'arrêt d'un traitement, plutôt que d'imposer aux médecins d'obtenir un consentement avant d'agir. Les implications de ce changement sont particulièrement troublantes quand le patient inapte ne dispose pas d'un réseau de parents ou d'amis ayant les ressources financières nécessaires pour entreprendre une action en justice, ce qui pourrait entraîner un procès sur le degré de soins fournis. En outre, il n'est pas évident, selon l'approche proposée, qu'un médecin puisse arrêter un traitement de maintien des fonctions vitales sans supervision judiciaire en l'absence d'action engagée par la famille ou les amis d'un patient.

L'une des principales motivations de la législature en adoptant la HCCA était de simplifier la loi régissant le traitement des patients inaptes. La loi HCCA établit des règles claires exigeant un consentement avant le début d'un traitement, identifiant qui peut consentir pour le compte d'un patient incapable, énonçant les critères sur la base desquels un consentement peut être accordé ou refusé, et créant un organisme spécialisé pour résoudre les litiges. Le cadre légal de la loi HCCA est utilisé depuis 17 ans pour résoudre les litiges de fin de vie en Ontario. Je serais réticente à bloquer le recours à un système établi et à renvoyer ces questions devant les tribunaux. »

Il est donc largement admis que les tribunaux d'arbitrage présentent de grands avantages par rapport aux tribunaux habituels. Dans un discours récent, la Juge en chef de la CSC a indiqué que: ${ }^{9}$

« Les tribunaux d'arbitrage fournissent des solutions spécialisées et techniques dans différentes situations. Ils permettent d'innover et offrent plus de souplesse et d'efficacité dans l'application des programmes et stratégies gouvernementales. Ils offrent aussi un forum rapide et informel pour la tenue d'audiences publiques, minimisant ainsi le temps et les coûts liés à la résolution des contentieux devant des tribunaux ordinaires. »

En conséquence, la procédure du CCB adoptée par l'Ontario et visant la résolution des conflits relatifs aux soins de santé représente une amélioration par rapport aux processus en vigueur dans les autres provinces ou territoires, lesquels reposent tous sur le jugement des cours. La procédure du CCB est moins formelle que celle d'une cour et est donc probablement moins angoissante et intimidante pour les parties concernées. Le CCB n'a pas, non plus, l'obligation de suivre des règles aussi strictes que les tribunaux classiques en matière de preuves. De telles règles peuvent donner naissance à un processus plus formel et technique. ${ }^{10}$ Enfin, c'est aux professionnels de la santé et non au mandataire d'entamer les démarches auprès du CCB. Ainsi, c'est sur le professionnel que repose la charge de démonter que le mandataire ne s'est pas conformé à l'article 21 de la loi HCCA de l'Ontario. Hawryluck et 
coll. préconisent que les mandataires s'adressent au tribunal pour demander une injonction, leur imposant alors le fardeau de la preuve pour l'obtention d'un jugement favorable. Une telle démarche représenterait un obstacle important pour les mandataires n'ayant pas les moyens financiers et émotionnels suffisants pour recourir aux tribunaux.

Bien qu'aucun tribunal ne soit parfait, nous sommes d'avis que les avantages de la procédure de résolution des conflits appliquée par le CCB dépassent largement ses faiblesses et que les autres juridictions devraient s'inspirer de ce que fait l'Ontario afin de réformer leurs législations sur le consentement aux soins de santé, et créer des tribunaux d'arbitrage pour la résolution des conflits en matière de consentement et d'aptitude à consentir.

Hawryluck et coll. concluent que « la décision dans le cas Rasouli doit être interprétée comme une invitation pour le secteur des soins intensifs à réfléchir et mieux définir son rôle dans les soins aux patients. Nous devons surtout expliquer aux patients et aux mandataires si nous sommes ou non capables de les aider, peu importe ce que nous souhaitons faire en tant que pourvoyeurs de soins de santé. » Notre point de vue est que la décision dans le cas Rasouli doit surtout être interprétée comme un appel aux Canadiens afin qu'ils exigent de leurs divers gouvernements une réforme de la loi afin de s'assurer que les médecins ne possèdent pas (ou ne croient pas avoir) l'autorité de maintenir ou d'interrompre unilatéralement un traitement visant le maintien de la vie. Comme cela a été démontré lors du débat en Ontario, les éléments primordiaux devraient être le respect de l'autonomie du patient et une présomption réfutable que les mandataires sont plus aptes que les médecins intensivistes à déterminer ce qui s'inscrit dans le meilleur intérêt de leurs êtres chers. La présomption initiale doit reconnaître aux patients ou à leurs mandataires l'autorité nécessaire à la prise de décision; les litiges étant résolus par un organisme de type CCB.
Conflicts of interest None declared.

Conflit d'intérêt Aucun déclaré.

\section{References}

1. Hawryluck L, Baker AJ, Faith A, Singh JM. The future of decision-making in critical care after Cuthbertson v. Rasouli. Can J Anesth 2014; 61: this issue. DOI:10.1007/s12630-014-0215-9.

2. Health Care Consent Act, s 37. Available from URL: http://www. e-laws.gov.on.ca/html/statutes/english/elaws_statutes_96h02_e. htm (accessed July 2014).

3. Cuthbertson v Rasouli, 2013 SCC 53, para 98. Available from URL: http://scc-csc.lexum.com/scc-csc/scc-csc/en/item/13290/ index.do (accessed July 2014).

4. Cuthbertson v Rasouli, 2013 SCC 53, para 101. Available from URL: http://scc-csc.lexum.com/scc-csc/scc-csc/en/item/13290/ index.do (accessed July 2014).

5. M. (A.) v Benes (1999), 46 O.R. (3d) 271, para 46. Available from URL: http://caselaw.canada.globe24h.com/0/0/ontario/court-ofappeal-for-ontario/1999/11/10/m-a-v-benes-1999-3807-on-ca.shtml (accessed July 2014).

6. Consent and Capacity Board. Available from URL: http://www. ccboard.on.ca/scripts/english/accountability/index.asp (accessed July 2014).

7. Healthcare Consent Quality Collaborative. "End of Life Cases". Available from URL: http://consentqi.ca/law/overview-2/end-oflife-cases/ (accessed July 2014).

8. Cuthbertson v Rasouli, 2013 SCC 53, paras 114 and 115. Available from URL: http://scc-csc.lexum.com/scc-csc/scc-csc/ en/item/13290/index.do (accessed July 2014).

9. Right Honourable Beverley McLachlin, Chief Justice of Canada "Administrative Tribunals and the Courts: An Evolutionary Relationship", 6th Annual Conference of the Council of Canadian Administrative Tribunals (May 27, 2013). Available from URL: http://www.scc-csc.gc.ca/court-cour/judges-juges/ spe-dis/bm-2013-05-27-eng.aspx\#fnb2 (accessed July 2014).

10. Section 15 of the Statutory Powers Procedure Act, R.S.O. 1990, c.22. Available from URL: http://www.e-laws.gov.on.ca/html/ statutes/english/elaws_statutes_90s22_e.htm (accessed July 2014). 